\title{
Dermoid cyst of the spermatic cord: a rare cause of benign inguinal lump
}

\author{
Muhammad Z. Aslam, MRCS, Ferhad Kheradmund, AFRCS, Nilay S. Patel, MRCS,; \\ Gareth Turner, FRCPath; ${ }^{\dagger}$ David Cranston, FRCS*
}

\section{Abstract}

Dermoid cyst of the spermatic cord is an extremely rare pathology seen at the urological clinic. We present the case of a 26-year-old man who presented with a groin lump, thought to be a lipoma or a hydrocoele of the cord. The final histopathological analysis established the diagnosis of a dermoid cyst.

Can Urol Assoc J 2009;3(4):E29-E30

\section{Case report}

A 26-year-old man presented to our urology clinic with a history of a mass in the right groin, which had been present for as long as he could remember. More recently he found this mass increasingly uncomfortable and felt that it interfered with his job. He was an otherwise healthy with no history of cryptorchidism or inguinal hernias.

On examination his abdomen was soft and nontender. There were no hernias or cough impulses in the inguinal region. Both testes were normal. In the right inguinal canal there was a 6-cm nontender fluctuant lump that was slightly transilluminable. An ultrasound showed the presence of a right groin mass consisting of 2 separate large cystic lesions with internal echoes within the inguinal canal. These measured $8 \times 2.5 \mathrm{~cm}$ and $10 \times 4.5 \mathrm{~cm}$. There were no reported sonographic features to suggest an underlying malignancy. The patient underwent an exploration of the right spermatic cord with the differential diagnosis of a lipoma or an encysted hydrocoele of the cord.

During surgery we noted 2 soft masses, each the size of a small orange, within the inguinal canal. They appeared separate from the cord, and we felt they contained fat. We excised both masses (Fig. 1 and Fig. 2).

Multiple histopathological sections from the cord lesions showed thin-walled cystic structures containing inspissated keratin and lined by mature keratinising squamous epithelium. The wall contained skin appendage structures with both eccrine glands and sebaceous glands. There was no evidence for dysplasia or malignancy in the lining of the cyst. The surrounding fatty connective tissue contained no residual or atrophic testicular tissue. The histological appearances suggested a dermoid cyst (Fig. 3). The patient had an uneventful recovery.

\section{Discussion}

Patients with groin swellings can present to the urologist in the outpatient clinic. Though the most common diagnosis is an inguinal hernia, other causes of groin swellings include femoral hernias, hydrocoeles, undescended testis, enlarged lymph nodes, lipoma, femoral artery aneurysm, saphena

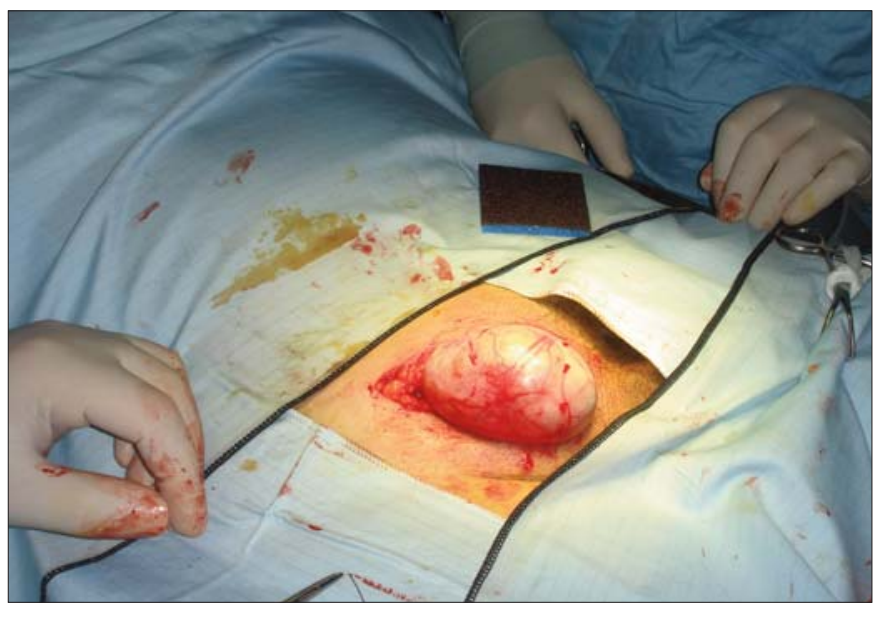

Fig. 1. Dermoid cysts of the cord delivered outside the inguinal wound.

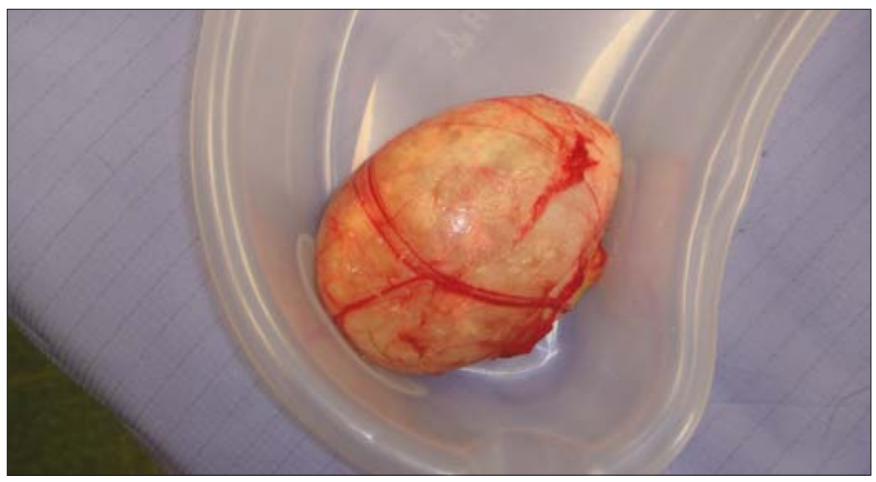

Fig. 2. Complete excision of the cysts. 
Aslam et al.

varix and varicocele. Rarer causes include inguinal endometriosis ${ }^{1}$ in women and thrombophlebitis of the long saphenous vein. ${ }^{2}$

A dermoid cyst of the spermatic cord is an unusual cause of groin swelling, with 8 cases reported to date. Dermoid is a loose term given to cysts lined by squamous epithelium occurring in various parts of the body. Dermoid cysts in and beneath the skin occur mostly on the face, neck or scalp. Dermoid cysts can also be intracranial, intraspinal or perispinal. Intra-abdominal dermoid cysts can also occur on the ovary or omentum.

Dermoid cysts occur when skin and skin structures become trapped during fetal development. Histogenetically, dermoid cysts are a result of the sequestration of skin along the lines of embryonic closure. Histologically the wall of a dermoid cyst consists of all components of the skin, including hair follicles, hair, sweat and sebaceous glands. ${ }^{3}$

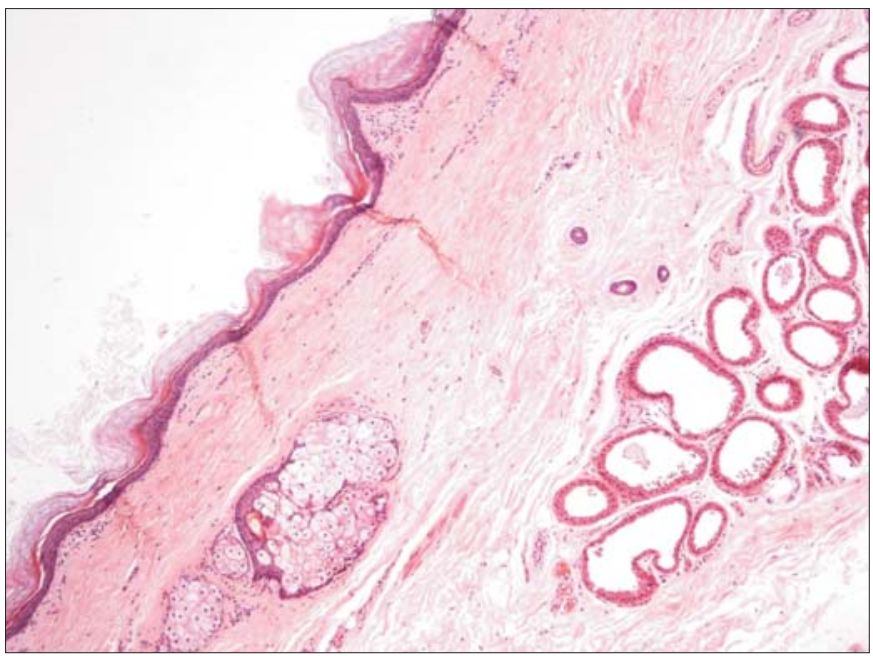

Fig. 3. Histopathological features suggestive of a dermoid cyst (hematoxylin and eosin stain, original magnification $\times 100$ ).
Dermoid cysts of the spermatic cord are not thought to be painful, ${ }^{4}$ though they have been known to mimic an incarcerated inguinal hernia. ${ }^{5,6}$ They have also been reported to occur in conjunction with an ipsilateral paratesticular epidermoid cyst. ${ }^{7}$ Dermoid cysts are always benign lesions. No case of malignant transformation has been reported. In symptomatic cases, surgical excision remains the only treatment option.

\section{Conclusion}

A dermoid cyst is a rare, benign cause of masses in the groin. The diagnosis may be suspected on ultrasound, but requires surgical excision and histopathological confirmation.

From the Departments of *Urology and TPathology, Oxford Radcliffe Hospitals, Oxford, UK

This paper has been peer reviewed.

Competing interests: None declared.

\section{References}

1. Jimenez M, Miles RM. Inguinal endometriosis. Ann Surg 1960;151:903-11.

2. Johnstone JM, Rintul RF. Unusual cause of inguinal mass. BMJ 1970;2:179.

3. Israel MS. Development of tumors and tumorl-ike lesions. In: IB Walter, MS. Israel, editors. General Pathology, 6th ed. Edinburgh (UK): Churchill Livingstone; 1987. pp. 370-86.

4. Ghosh DP. Dermoid cyst of the spermatic cord. J Indian Med Assoc 1980;74:18-9.

5. Leeming R, Olsen M, Ponsky JL.Inguinal dermoid cyst presenting as an incarcerated inguinal hernia. J Paediatric Surg 1992;27:117-8.

6. Brightmore T. Dermoid cyst of the inguinal canal simulating a strangulated inguinal hernia. $\mathrm{Br} J$ Clin Pract 1971;25:191.

7. Wegner HEH, Herbest H, Dieckmann K.P. Paratesticular epidermoid cyst and ipsilateral spermatic cord dermoid cyst: case report and discussion of pathogenesis, diagnosis and treatment. J Urol 1994;152:2101-3.

Correspondence: Dr. Muhammad Z. Aslam, 7 Hillbrook Cres., Ingleby Barwick, Stockton-on-Tees TS17 5BN UK; mzaslam77@yahoo.com 